\title{
Wie viel Training brauchen Arbeitslose?
}

\section{Carina Steckenleiter}

\section{Relevanz}

Die Bekämpfung der Arbeitslosigkeit ist eines der wichtigsten Ziele der Wirtschaftspolitik. Jährlich wenden Industrienationen beträchtliche Summen an Geldern für Arbeitsmarktprogramme auf. Laut einer vergleichenden Aufstellung der OECD entsprachen im Jahr 2015 die Ausgaben für aktive Arbeitsmarktprogramme in Deutschland beispielsweise $0,6 \%$ des Bruttoinlandsprodukts (BIP) und in Frankreich $1 \%$ des BIPs. Vor dem Hintergrund dieser hohen Kosten sind belastbare Auswertungen, die die Wirkung der Programme evaluieren, von immenser Bedeutung. Die Autoren der vorliegenden Studie evaluieren dabei insbesondere den Effekt der Dauer eines Arbeitsmarktprogramms.

\section{Quelle}

Flores, Carlos A., Alfonso Flores-Lagunes, Arturo Gonzalez, und Todd C. Neumann (2012), Estimating the Effects of Length of Exposure to Instruction in a Training Program: The Case of Job Corps, Review of Economics and Statistics 94(1), 153-171.

Arbeitsmarktprogramme sind ein etabliertes Instrument zur Arbeitsmarktintegration von arbeitslosen Personen. Es erscheint plausibel, dass neben der Tatsache, ob jemand an einem Programm teilgenommen hat, auch die Programmdauer und somit die Intensität den späteren Arbeitsmarkterfolg beeinflussen. So

C. Steckenleiter $(\bowtie)$

Universität St.Gallen, St.Gallen, Schweiz

E-Mail: carina.steckenleiter@unisg.ch 
ist es einerseits vorstellbar, dass sich der Nutzen eines Programms erst ab einer bestimmten Dauer der Programmteilnahme materialisiert. Andererseits erscheint es ebenso plausibel, dass ab einer bestimmten Programmdauer kein zusätzlicher Nutzen für den Teilnehmer entsteht. Darüber hinaus könnte es sein, dass verschiedene Gruppen wie zum Beispiel jüngere und ältere Teilnehmer unterschiedlich reagieren. All diese Informationen sind für politische Entscheidungsträger wichtig. Sie können dazu dienen, Programme besser auf die Bedürfnisse der Teilnehmer anzupassen und Steuergelder kosteneffizient einzusetzen. Die Arbeit von Flores, Flores-Lagunes, Gonzalez, und Neumann ist eine der ersten Studien, die den Wirkungszusammenhang zwischen Programmlänge und späterem Arbeitsmarkterfolg erforscht.

Die Autoren analysieren am Beispiel des Job Corps Programms in den USA, welchen Effekt die Länge der Programmteilnahme auf den Arbeitsmarkterfolg hat. Das Job Corps Programm existiert seit 1964 und kann landesweit an mehr als 120 Zentren absolviert werden. Es bietet unter anderem Berufsausbildung, Kurse zur Stärkung der Sozialkompetenz und verschiedene Komponenten von Schulbildung wie zum Beispiel Mathematikkurse oder vorbereitende Kurse zum Absolvieren des High-School Abschlusses an. 16- bis 24-jährige können bei Erfüllung verschiedener Kriterien am Programm teilnehmen. Zu diesen zählen unter anderem Armutsstatus, das Leben in einem schwierigen Umfeld, ein Schulabbrecher zu sein, oder das Benötigen von weiterer Bildung/Ausbildung. Der typische Programm-Teilnehmer gehört zu $70 \%$ einer Minderheit an, ist 18 Jahre alt und hat in $75 \%$ der Fälle die High-School abgebrochen. Etwa 60.000 junge Erwachsene beginnen jedes Jahr das Programm. Besonders am Job Corps Programm ist, dass teilnahmeberechtigte Bewerber zufällig zur Teilnahme ausgewählt wurden. Dies erlaubt die Evaluation der Programmeffekte mit gängigen ökonometrischen Methoden.

Der typische Programm-Teilnehmer gehört zu $70 \%$ einer Minderheit an, ist 18 Jahre alt und hat zu $75 \%$ die High-School abgebrochen.

Gemeinsam mit einem Berater entwickelt jeder Teilnehmer einen Programmplan, welcher von den eigenen Bedürfnissen und Präferenzen und den Charakteristika des jeweiligen Programmcenters abhängt. Die Teilnahmedauer einer Person setzt sich somit aus institutionellen Gegebenheiten sowie persönlichen Entscheidungen zusammen. Die durchschnittliche Teilnahmedauer entspricht 30,4 Wochen, somit in etwa 7,5 Monate.

Die Autoren der Studie messen den Arbeitsmarkterfolg anhand von zwei Indikatoren: Zum einen betrachten sie das durchschnittliche wöchentliche Einkommen der Teilnehmer 48 Monate nach zufälliger Programmzuteilung, und zum anderen das durchschnittliche wöchentliche Einkommen 1 Jahr nach Programm- 
ende. Beide Indikatoren ermitteln sie sowohl absolut als auch in Differenz zum Einkommen, welches die Individuen vor der Programmteilnahme verdient haben.

Abb. 1 zeigt die Ergebnisse der Studie zum Effekt der Programmdauer auf das durchschnittliche wöchentliche Einkommen 1 Jahr nach Programmende für verschiedene Gruppen. Die Ergebnisse werden einmal für die gesamte Stichprobe, aber auch zusätzlich für die Teilstichproben Männer, Frauen, Afroamerikaner, Weisse sowie Hispanics gezeigt. Die Abbildung beschreibt den marginalen Effekt einer zusätzlichen Woche Programmteilnahme auf das durchschnittliche Wocheneinkommen ein Jahr nach dem Ende der Teilnahme (in Differenzen). Punkte auf der schwarzen Linie oberhalb der Null-Dollar-Grenze geben an, um wie viel das Einkommen bei einer Verlängerung der Programmteilnahme um eine zusätzliche Woche steigen würde. Die Grafik zeigt, dass dies für alle Gruppen zumeist der Fall ist. Jedoch ist auch klar zu erkennen, dass der Effekt einer zusätzlichen Woche auf das Einkommen mit zunehmender Programmdauer kleiner wird. Jede Schätzung weist eine Unsicherheit auf, welche in der Abbildung durch die gestrichelten Linien (Konfidenzintervalle) dargestellt wird. Wenn ein geschätzter

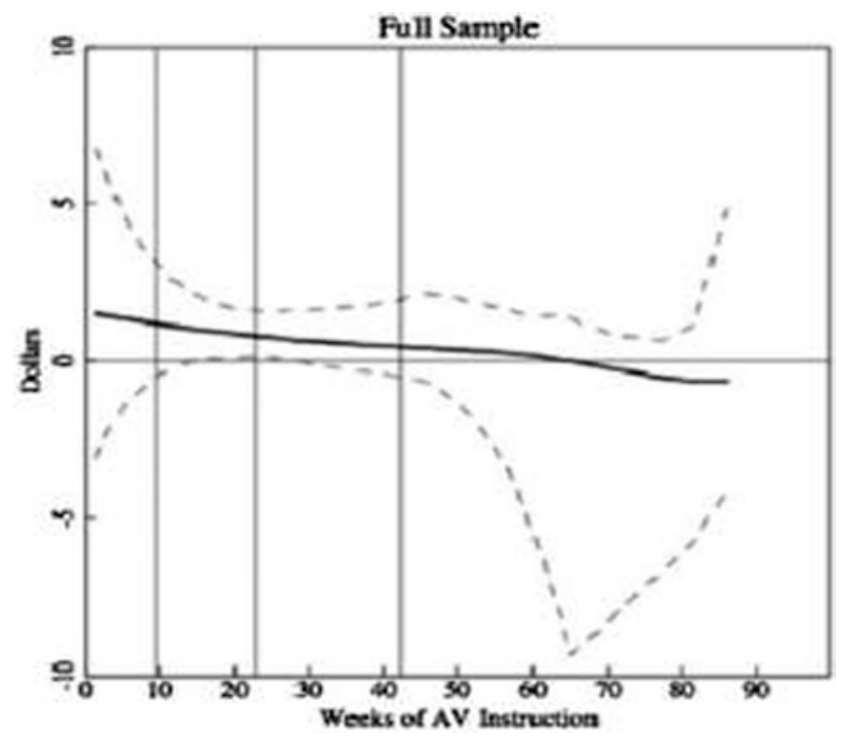

Abb. 1 Effekt einer zusätzlichen Woche Programmteilnahme auf das durchschnittliche wöchentliche Einkommen $1 \mathrm{Jahr}$ nach Programmende in Differenz zum Einkommen vor Programmteilnahme. (Quelle: Flores et al. 2012, S. 167) 
Effekt positiv und statistisch signifikant von Null verschieden ist, befinden sich alle Werte des Intervalls oberhalb der Nullgrenze. Dies ist z. B. für die gesamte Stichprobe bis zu einer Programmdauer von ungefähr 31 Wochen der Fall.

Der durchschnittliche marginale Ertrag einer weiteren Woche Programmteilnahme wird auf 2,1 US-Dollar geschätzt, das sind 1,8 \% des Einkommens vor der Programmteilnahme. Bei einer Programmdauer von 10 Wochen würde eine Verlängerung um eine Woche das Wocheneinkommen um ca. 4 US-Dollar steigern. Der marginale Ertrag einer verlängerten Teilnahme von 23 auf 24 Wochen entspricht in etwa noch der Hälfte der Einkommenssteigerung von 10 auf 11 Wochen Teilnahme.

Vergleicht man die einzelnen Abbildungen miteinander, stellt man teilweise deutliche Unterschiede in den Einkommenszuwächsen fest, welche verschiedene Gruppen bei einer Verlängerung des Programms um eine Woche erzielen könnten. Wenn man beispielsweise Männer und Frauen über die Programmwochen 1-42 hinweg vergleicht, lassen sich interessante Unterschiede feststellen. Der durchschnittliche marginale Ertrag einer weiteren Woche Programmteilnahme beträgt für Männer 3,1 US-Dollar pro Woche, für Frauen dagegen nur 1,3 US-Dollar.

Die Studie vergleicht zudem die Auswirkungen auf das durchschnittliche Wocheneinkommen 48 Monate nach Programmzuteilung bzw. 1 Jahr nach Programmende. Die Forscher zeigen, dass der geschätzte Einkommenszuwachs von einer Woche mehr Training 1 Jahr nach Programmende höher ist. So beträgt der geschätzte durchschnittliche marginale Ertrag einer zusätzlichen Woche Programmteilnahme 1 Jahr nach Programmende 2,1 US-Dollar, während dieser 48 Monate nach Programmzuteilung auf 0,8 US-Dollar geschätzt wird (beides in Differenz zu vor Programmbeginn erzieltem Einkommen). Die Autoren schlussfolgern daraus, dass sogenannte „Lock-in-Effekte“ im Job-Corps-Programm von Bedeutung sind. Als „Lock-in-Effekte“ bezeichnen Arbeitsmarktökonomen negative Beschäftigungseffekte unmittelbar nach Beginn des Arbeitsmarktprogramms, welche dadurch entstehen, dass Teilnehmer weniger Zeit zur Stellensuche aufwenden können.

Über die gesamte Programmdauer betrachtet ist der durchschnittliche Einkommenszuwachs aus einer Verlängerung der Programmteilnahme um 1 Woche für Männer zweimal so hoch wie für Frauen.

Insgesamt zeigt die Studie einen positiven Zusammenhang zwischen einer längeren Partizipationsdauer und dem nachher erzielten wöchentlichen Ein- 
kommen auf. Die Wirkung einer Verlängerung um eine Woche nimmt jedoch mit der Dauer des Programms ab. Interessanterweise fällt der Nutzen sehr unterschiedlich für verschiedene Gruppen aus. Männer profitieren im Durchschnitt doppelt so stark wie Frauen. Die Studie zeigt somit zum einen auf, dass die Dauer der Programmteilnahme ein wichtiger Baustein für Arbeitsmarktprogramme ist, und zum anderen, dass es beträchtliche Unterschiede zwischen verschiedenen demographischen Gruppen gibt.

Open Access Dieses Kapitel wird unter der Creative Commons Namensnennung 4.0 International Lizenz (http://creativecommons.org/licenses/by/4.0/deed.de) veröffentlicht, welche die Nutzung, Vervielfältigung, Bearbeitung, Verbreitung und Wiedergabe in jeglichem Medium und Format erlaubt, sofern Sie den/die ursprünglichen Autor(en) und die Quelle ordnungsgemäß nennen, einen Link zur Creative Commons Lizenz beifügen und angeben, ob Änderungen vorgenommen wurden.

Die in diesem Kapitel enthaltenen Bilder und sonstiges Drittmaterial unterliegen ebenfalls der genannten Creative Commons Lizenz, sofern sich aus der Abbildungslegende nichts anderes ergibt. Sofern das betreffende Material nicht unter der genannten Creative Commons Lizenz steht und die betreffende Handlung nicht nach gesetzlichen Vorschriften erlaubt ist, ist für die oben aufgeführten Weiterverwendungen des Materials die Einwilligung des jeweiligen Rechteinhabers einzuholen.

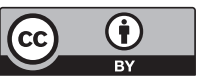

